ISBN 978-981-14-1684-2

Proceedings of 2019 the 9th International Workshop on Computer Science and Engineering

(WCSE 2019 SUMMER)

Hong Kong, 15-17 June, 2019, pp. 72-77

doi: 10.18178/wcse.2019.06.012

\title{
A Cluster-based Sample Selection Strategy for Biological Event Extraction
}

\author{
Yang $\mathrm{Lu}^{1,2}$, Xiaolei $\mathrm{Ma}^{1,2}$, Yinan $\mathrm{Lu}^{* 1}$ \\ ${ }^{1}$ College of the Computer Science and Technology, Jilin University \\ Changchun, 130000, Jilin, China \\ ${ }^{2}$ Library, Inner Mongolia University for Nationalities, Tongliao, 028000, Inner Mongolia, China
}

\begin{abstract}
Biological Event Extraction is an important and difficult task, whose purpose is to obtain biomedical knowledge from biomedical literature by identifying biomedical entities and extracting their complex relations from the texts. However, biological events are highly complex and the annotated biomedical corpus is highly imbalanced, which affects the performance of classifier. In this paper, we develop a new semi-supervised biomedical event extraction method based on pairwise model. Firstly, we use initial trained classifier to predict trigger-argument pairs of unlabeled data in sentences. Secondly, we have presented a sentence representation method that combines dependent path by word embedding from biomedical text. Then we fed it to cluster to filter noise samples by a sample selection strategy, and the rest of samples are added to the training dataset to balance dataset. Experimental results on BioNLP-ST GENIA corpus show that the proposed method demonstrates its effectiveness and achieves better performance.
\end{abstract}

Keywords: Biological Event Extraction, Sentence Representation, Cluster.

\section{Introduction}

With the exponential growth of electronic biological literature resources, it becomes harder than ever for researchers to read and extract relevant knowledge from unstructured or semi-structured literature. To address these problems, natural language processing techniques are applied to biological literatures to facilitate the domain knowledge automatic extraction process, i.e. relationships between genes and disease [1], protein and protein interactions (PPI) [2] and some complex biological information extraction [3].

The BioNLP Shared Tasks (BioNLP-ST) focuses on extracting such complex biological process relations called biological event from biomedical texts. The biological event describes a detailed behavior of bio-molecules, usually consisting of "event type", "trigger" and one or more "arguments". Table 1 shows argument of event and three event types: Simple event class (SVT), Binding event class (BIND) and Regulation event class (REG). Fig.1 shows an example of the event, two complex biological event in a sentence "We studied a putative cause for absent IRF-4 expression in leukemia cells and first focused on genetic aberrations of the promoter." can be annotated as follows:

1) Event E1: (Event Type: Negative_regulation, Trigger: absent, Theme: E2)

2) Event E2: (Event Type: Gene_expression, Trigger: expression, Theme: IRF-4)

Generally, the biological event extraction task can be categorized into three steps, i.e., 1) event trigger identification, 2) the edge detection, and 3) assigns the arguments on the basis of the first step. The event

\footnotetext{
* Corresponding author. Tel.: +8613843100175

E-mail address: 97088340@qq.com (Y.Lu), 342483801@qq.com (X. Ma), luyn@jlu.edu.cn (Y. Lu)
} 
trigger is an anchor that indicate the occurrence of event, and the event arguments are "cause" and "theme" of the biological event. The method of biological event extraction is called pipeline model.

Table 1: Event Class, Event type and their arguments of GENIA task

\begin{tabular}{|c|c|c|c|}
\hline $\begin{array}{c}\text { Event } \\
\text { class }\end{array}$ & Event type & Primary Argument & $\begin{array}{c}\text { Secondary } \\
\text { Argument }\end{array}$ \\
\hline \multirow{4}{*}{ SVT } & Gene_expression & Theme(P) & \\
\cline { 2 - 4 } & Transcription & Theme(P) & \\
\cline { 2 - 4 } & Localization & Theme(P) & AtLoc, ToLoc \\
\cline { 2 - 4 } & Protein_catabolism & Theme(P) & Site \\
\cline { 2 - 4 } & Phosphorylation & Theme $(\mathrm{P})$ & Site+ \\
\hline \multirow{3}{*}{ BIND } & Binding & Theme $(\mathrm{P})+$ & Site, Csite \\
\hline \multirow{3}{*}{ REG } & Regulation & Theme(P/E), Cause $(\mathrm{P} / \mathrm{E})$ & Site, Csite \\
\cline { 2 - 4 } & Positive_regulation & Theme(P/E), Cause $(\mathrm{P} / \mathrm{E})$ & Site, Csite \\
\cline { 2 - 4 } & Negative_regulation & Theme(P/E), Cause $(\mathrm{P} / \mathrm{E})$ & \\
\hline
\end{tabular}

$P$ represents protein, E represents Event

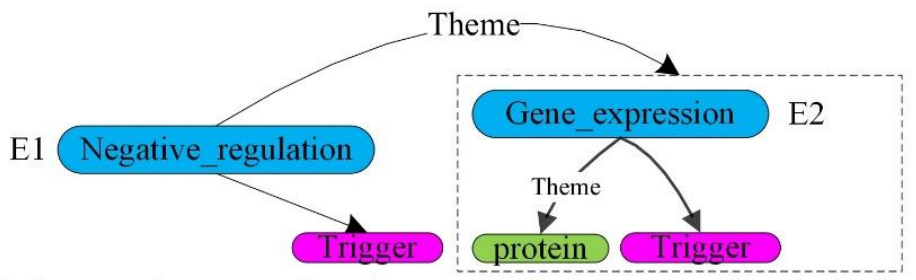

We studied a putative cause for absent IRF-4 expression in leukemia cells and first focused on genetic aberrations of the promoter.

Fig. 1: Structured representation of biological event

Turku [4] and EVEX [5] systems in the event extraction task had achieved excellent results by using Pipeline model. Pyysalo et al. [6] used pipeline model with SVM and designed features to conduct event triggers and arguments identification separately for event extraction. In their study, they revealed that more than $60 \%$ of event extraction errors were related to incorrect event trigger extraction. That is to say, if a mistake is made in the first step in pipeline model, it will propagate to the next step, causing a cascade of errors. Therefore, how to efficiently reduce cascading error while improving event extraction accuracy has become a huge challenge.

The Joint model solved cascade of errors occurred in Pipeline model. Riedel and McCallum [7] used the dual-decomposition method to detect triggers and arguments. The study [8] used sentence boundaries to perform joint inference by extracting event mentions and entity mentions together for improving the study [7].

The Pairwise model [9] combined the characteristics of Pipeline model and Joint model: it integrated the first two step of Pipeline model, and in post-processing, it used a small amount of inference, which leading to higher accuracy than the Pipeline model and a faster execution time than the Joint model.

In this paper, we develop a new semi-supervised biomedical event extraction method based on Pairwise model, which can effectively avoid cascading errors. First, we use initial trained classifier to predict triggerargument pairs of unlabeled data in sentences; then, the dependency paths between these triggers and corresponding parameters are extracted and represented by word vector as input of cluster; finally, a sample selection strategy based on cluster are applied to filter noise sample.

\section{Methods}

Our main work proceeded as follows. First, we trained dependency-based word embedding to represent the dependency path between triggers and corresponding arguments from all available PubMed abstracts. Then, we used a sample selection strategy based on cluster are applied to filter noise sample from unlabeled sample. The event extraction process is summarized in Fig.2. 


\subsection{Text preprocessing}

Text preprocessing is the critical step and an important task in natural language processing. In the process, some words and sentences are identified, and some useless symbols are removed, such as publication date, publishing company and authors, etc. We used the Natural language toolkit (nltk.org) and Charniak-Johnson parser with McClosky's et al. (2011) [10] biomedical parsing model in biomedical event extraction for text preprocessing.

After text preprocessing, features designed based on TEES [11] are used in our system. In fact, there are many features including a lot of redundancy or invalid information, therefore, it is necessary to select features to reduce the dimension of input data, which also helps to shorten training time and obtain effective models. Features representing a word for a candidate entity or a pair of words (trigger and argument) for event extraction are described as follows:

- Token features: include current token text, spelling features, POS-tag includes the POS of the current token and, character $n$-grams $(n=\{1,2,3\})$, the stem of token, the lexical string of token, the lemma of token.

- Sentence features: the number of candidate entities, bag-of-words, whether the candidate trigger word contains a symbol,

- Sentence dependency features: dependency chain features and the shortest dependency path features between trigger and entity.

- External resource features: Wordnet hypernyms

\subsection{Sentence representation}

The sentence is the shortest dependency path between trigger and argument of candidate pair from prediction. Let the threshold $\delta$, if the length of the sentence is less than threshold $\delta$, extend the sentence length to given threshold. We expand the shortest dependency path by adding the neighboring node of each node in this path and then candidate sentences set is obtained. Sentences are represented in the form of vectors. Therefore, word $2 \mathrm{vec}$ is utilized, which analyzes semantic and syntactic information among words. We train a word vector model with $30 \mathrm{~Gb}$ biomedical scientific literature from PubMed abstracts. Each sentence $\mathrm{s}$ is represented as a list $\left\{\mathrm{w}_{1}, \mathrm{w}_{2}, \cdots, \mathrm{w}_{\mathrm{n}}\right\}$, where $\mathrm{w}_{\mathrm{i}}$ is the vector of the word, and convert the sentence with word $2 \mathrm{vec}$ items into a set of sentence vectors.

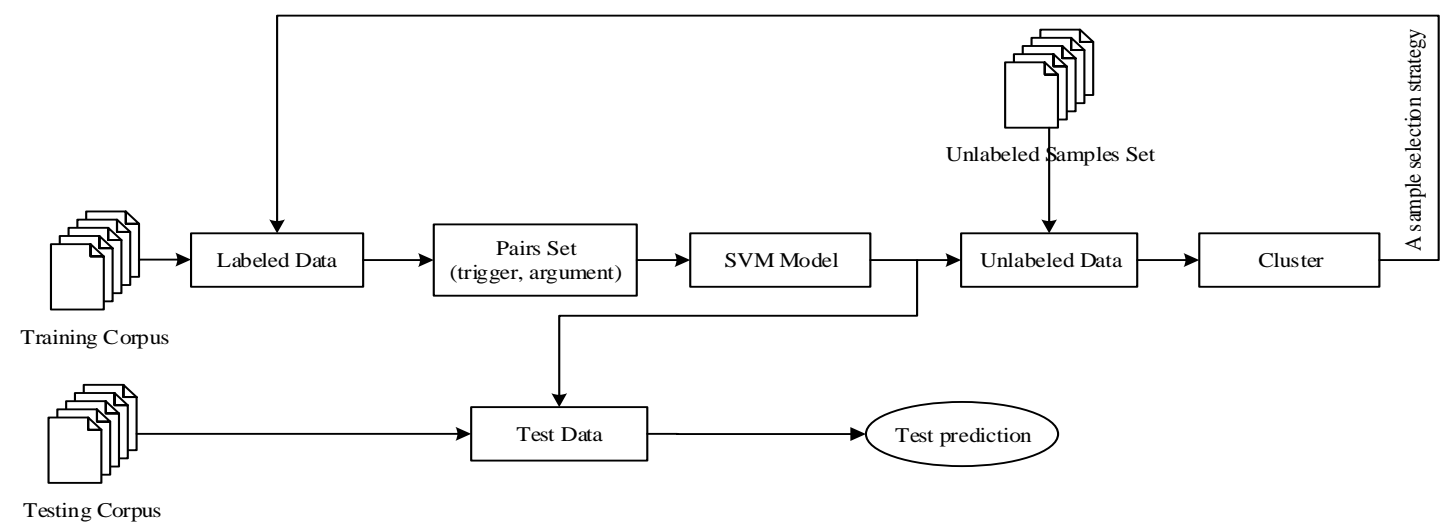

Fig. 2: The framework of the proposed method.

\subsection{Intelligent clustering analysis}

In this study, we select both the most certain and uncertain samples. For most uncertain samples, which is to select sentences of candidate pairs that are the least of class labels and close to the cluster centroid in each cluster. Let $D S_{i}=\left\{S_{i 1}, S_{i 2}, \cdots, S_{i h}\right\}$ be the set of sentences found in the same cluster $C_{i}$, the sentences that are the least of class labels is defined as follows:

$$
\operatorname{LS}\left(S_{\mathrm{i}}\right)=\left\{\mathrm{S}_{\mathrm{i}}^{\mathrm{p}} \in \mathrm{C}_{\mathrm{i}} \mid \forall \mathrm{q} \in[1, \cdots, \mathrm{k}], \mathrm{p} \neq \mathrm{q}, \operatorname{num}\left(\mathrm{S}_{\mathrm{i}}^{\mathrm{p}}\right) \leq \operatorname{num}\left(\mathrm{S}_{\mathrm{i}}^{\mathrm{q}}\right)\right\}
$$

Where num $\left(S_{i}^{p}\right)$ and num $\left(S_{i}^{q}\right)$ are the number of sentences with class label $p$ and $q$ respectively. For each cluster $C_{i}$, note that the samples with minority class labels could not represent the class of cluster. This 
means that this portion of minority samples are most uncertain. Especially those samples which close to the cluster's centroid. As expressed in Equation (4), the point (sentence) of distance close to the centroid $r_{i}$ less than the threshold $\mu$ will be selected.

$$
\operatorname{LD}\left(\mathrm{S}_{\mathrm{i}}\right)=\left\{\mathrm{S}_{\mathrm{ij}} \in \mathrm{LS}\left(\mathrm{S}_{\mathrm{i}}\right) \mid \operatorname{dist}\left(\mathrm{S}_{\mathrm{ij}}, \mathrm{r}_{\mathrm{i}}\right)<\mu, \forall \mathrm{j} \in\left[1, \cdots,\left|\mathrm{LS}\left(\mathrm{S}_{\mathrm{i}}\right)\right|\right]\right\}
$$

where $\left|\mathrm{LS}\left(\mathrm{S}_{\mathrm{i}}\right)\right|$ is the number of sentences that belong to the minority classification in cluster $\mathrm{C}_{\mathrm{i}}$. dist indicates the distance between the point (sentence) $S_{i j}$ and the centroid $r_{i}$. Finally, samples that belong to the minority class and close to the centroid are discarded because we think the samples are most uncertain sample.

We apply proposed method to improve event extraction performance by progressively selecting appropriate samples for model updating. In the process, we classification first and then cluster to avoid generating noisy samples. The Intelligent Cluster-based algorithm is presented in Algorithm 1.

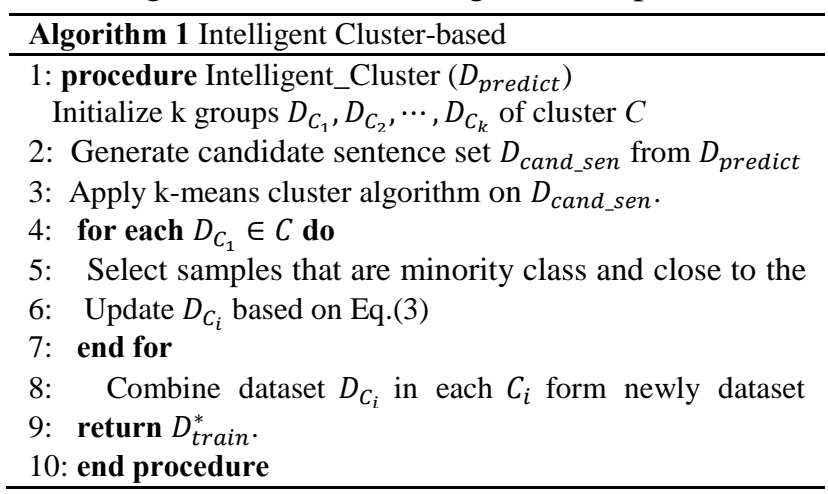

\section{Experiment and Results}

\subsection{Experimental setup}

We evaluate the performance of our proposed method and compare it with the results of other systems on BioNLP-ST GENIA Event Extraction 2011 (GE'11) and 2013 (GE'13) corpus. On the GENIA corpus, we combine training and development datasets for training, and the test set is used to test. We adopt $\mathrm{R}($ ecall $) / \mathrm{P}($ recision $) / \mathrm{F}$ (-score) as the evaluation criteria. The evaluation metric $\mathrm{R} / \mathrm{P} / \mathrm{F}$ is defined as below (3), where TP, FP and FN correspond to true positive, false positive and false Negative respectively.

$$
\mathrm{R}=\frac{\mathrm{TP}}{\mathrm{TP}+\mathrm{FN}}, \mathrm{P}=\frac{\mathrm{TP}}{\mathrm{TP}+\mathrm{FP}}, \mathrm{F}=\frac{2 * \mathrm{R} * \mathrm{P}}{\mathrm{R}+\mathrm{P}}
$$

Table 2: Results of the proposed method on GE'11 test set

\begin{tabular}{|l|c|c|c|}
\hline \multicolumn{1}{|c|}{ Event class } & R & P & F \\
\hline Gene expression & 73.15 & 84.94 & 78.61 \\
\hline Transcription & 51.72 & 72.00 & 60.20 \\
\hline Protein catabolism & 60.00 & 75.00 & 66.67 \\
\hline Phosphorylation & 56.22 & 83.20 & 67.10 \\
\hline Localization & 30.89 & 80.82 & 44.70 \\
\hline SVT & 63.50 & 83.06 & 71.97 \\
\hline BIND & 48.68 & 56.24 & 52.18 \\
\hline Regulation & 25.97 & 52.91 & 34.84 \\
\hline Positive regulation & 36.45 & 54.45 & 43.67 \\
\hline Negative regulation & 41.68 & 45.42 & 43.47 \\
\hline REG & 36.02 & 51.46 & 42.37 \\
\hline ALL TOTAL & 47.07 & 63.54 & 54.08 \\
\hline
\end{tabular}

\subsection{Results of the GE'11 shared task}

We compared the performance of our method with other systems to illustrate the excellence of the method in event classification. Moreover, Table 2 shows the results of each event on the GE'11 test set, where the purpose is to better illustrate which of our methods is more advantageous. As can been seen from Table 3, the precision and F-score of our approach received the highest score, and our approach also performs very well in extracting the binding events class. This result may be related to the new semisupervised method we proposed. These results prove that our approach with SVM and cluster is effective on the GE'11 test set. 
Table 3: Comparison with other systems on GE'11 test set

\begin{tabular}{|c|c|c|c|c|}
\hline System & SVT & BIND & REG & ALL \\
\hline UTurku & $68.22 / 76.47 / 72.11$ & $42.97 / 43.60 / 43.28$ & 38.72/47.64/42.72 & 49.56/57.65/53.30 \\
\hline MSR-NLP & 68.99/74.30/71.54 & $42.36 / 40.47 / 41.39$ & $36.64 / 44.08 / 40.02$ & $48.64 / 54.71 / 51.50$ \\
\hline Ours & $63.50 / 83.06 / 71.97$ & $48.68 / 56.24 / 52.18$ & $36.02 / 51.46 / 42.37$ & $47.07 / 63.54 / 54.08$ \\
\hline
\end{tabular}

\subsection{Results of the GE'13 shared task}

Unlike the GE'11 test data, the GE'13 test set contains only full text and does not include abstracts. We compared two best systems, TEES 2.1 and EVEX. Table 4 shows the results of each event on the GE'13 test set. From Table 5, we can see that there is no particular aspect of EVEX that is more effective on the GE'13, and the total F-score of EVEX is 2.73 less than our method. The result obtained from our method shows that our approach performed very well, with precision in every event class, it is 83.77, 45.77, 57.16 and 66.58 in SVT, BIND, REG and ALL events, respectively. Our recall rate in SVT and BIND are lower than those in other systems. The recall of our system is 71.20, while the recall of TEES2.1 and EVEX are 74.52 and 73.82, respectively. Nevertheless, our method performs well on the total precision and F-score, with values of 66.58 and 53.97, respectively. In particular, the total precision is almost 10 points higher than that of other systems. These results clearly demonstrate effectiveness of our method, which may be attributed to the intelligent clustering analysis.

Table 4: Results of our method on GE'13 test set

\begin{tabular}{|l|c|c|c|}
\hline \multicolumn{1}{|c|}{ Event class } & R & P & F \\
\hline Gene expression & 80.45 & 89.25 & 84.62 \\
\hline Transcription & 47.52 & 67.61 & 55.81 \\
\hline Protein catabolism & 57.14 & 66.67 & 61.54 \\
\hline Phosphorylation & 76.88 & 75.46 & 76.16 \\
\hline Localization & 30.30 & 75.00 & 43.17 \\
\hline SVT & 71.20 & 83.77 & 76.98 \\
\hline BIND & 39.04 & 45.77 & 42.14 \\
\hline Regulation & 17.36 & 49.50 & 25.71 \\
\hline Positive regulation & 34.60 & 61.97 & 44.41 \\
\hline Negative regulation & 39.16 & 51.50 & 44.49 \\
\hline REG & 33.28 & 57.16 & 42.07 \\
\hline ALL TOTAL & 45.38 & 66.58 & 53.97 \\
\hline
\end{tabular}

Table 5: Comparison with other systems on GE'13 test set

\begin{tabular}{|c|c|c|c|c|}
\hline System & SVT & BIND & REG & ALL \\
\hline TEES 2.1 & 74.52/77.73/76.09 & 42.34/44.34/43.32 & $33.08 / 44.78 / 38.05$ & 46.60/56.32/51.00 \\
\hline EVEX & $73.82 / 77.73 / 75.72$ & $41.14 / 44.77 / 42.88$ & $32.41 / 47.16 / 38.41$ & $45.87 / 58.03 / 51.24$ \\
\hline Ours & 71.20/83.77/76.98 & $39.04 / 45.77 / 42.14$ & 33.28/57.16/42.07 & $45.38 / \mathbf{6 6 . 5 8} / \mathbf{5 3 . 9 7}$ \\
\hline
\end{tabular}

The above experimental results provide strong evidence for our proposed method. Firstly, it can effectively alleviate data imbalance. Due to the limited training data, the problem of data imbalance is very serious. Therefore, we use cluster to iteratively increase samples to train data, which can alleviate the problem of data imbalance. Secondly, these experimental results clearly demonstrate the effectiveness of this method. However, there are still some shortcomings: 1) The large amount of computation in program operation results in high time complexity. 2) The features used in cluster computation need to be improved. 3) Single classifier has shortcomings in event extraction performance. In future work, we will try to combine multiple classifiers to improve the accuracy of biomedical event extraction, and improve the performance of classifiers by adding positive samples, removing redundant negative samples and features.

\section{Conclusions}

In this paper, a new semi-supervised event extraction system is introduced. Firstly, we use initial trained classifier to predict trigger-argument pairs of unlabeled data in sentences. Secondly, we have presented a sentence representation method that combines dependent path by word embedding from biomedical text. Then we fed it to cluster to filter noise samples by a sample selection strategy. Our method is an iterative process, which selects appropriate samples from unlabeled data for model updating to improve event extraction performance. Our method achieved some positive results by comparing other event extraction systems. Although numerous efforts are made, extracting complex events is still a difficult task. In the future, 
we will further optimize sample selection strategy based cluster to improve the effectiveness of the experiment.

\section{Acknowledgements}

This work was supported by Science and Technology Development Program of Jilin Province (No. 20180101054JC), Foundation Sciences of Inner Mongolia University for Nationalities (NMDYB17124, NMDYB17125).

\section{References}

[1] Zhang Y, Shen F, Mojarad M R, et al. Systematic identification of latent disease-gene associations from PubMed articles. PlOS One, 2018, 13(1): e0191568.

[2] Ashtiani M , Salehzadehyazdi A , Razaghimoghadam Z, et al. A systematic survey of centrality measures for protein-protein interaction networks. BMC Systems Biology, 2018, 12.

[3] Kim, Jin-Dong, et al. "Overview of BioNLP'09 shared task on event extraction." Proceedings of the Workshop on Current Trends in Biomedical Natural Language Processing: Shared Task. Association for Computational Linguistics, 2009.

[4] Björne, Jari, Filip Ginter, and TapioSalakoski. "University of Turku in the BioNLP'11 shared task." BMC bioinformatics 13.11 (2012): 1

[5] Hakala, Kai, et al. "EVEX in ST'13: Application of a large-scale text mining resource to event extraction and network construction." Proceedings of the BioNLP Shared Task 2013 Workshop. Association for Computational Linguistics, 2013.

[6] Pyysalo S , Ohta T , Miwa M , et al. Event extraction across multiple levels of biological organization. Bioinformatics, 2012, 28(18):i575-i581.

[7] Riedel, S., \& Mccallum, A. (2011) Fast and robust joint models for biomedical event extraction. Conference on Empirical Methods in Natural Language Processing (Vol.1-12, pp.1-12). Association for Computational Linguistics.

[8] Yang, B., \& Mitchell, T. (2016) Joint extraction of events and entities within a document context. 289-299.

[9] Liu X, Bordes A, Grandvalet Y. Extracting biomedical events from pairs of text entities. BMC bioinformatics 2015, 16(10): S8

[10] McClosky D, Surdeanu M, Manning CD. Event extraction as dependency parsing. Proceedings of the 49th Annual Meeting of the Association for Computational Linguistics: Human Language Technologies-Volume 1. Association for Computational Linguistics 2011: 1626-1635.

[11] Björne, Jari, and TapioSalakoski. "TEES 2.1: Automated annotation scheme learning in the BioNLP 2013 Shared Task." Proceedings of the BioNLP Shared Task 2013 Workshop. 2013. 